\title{
Corruzione morale e virtù nel pensiero di Posidonio. Per il riesame di alcuni frammenti
}

\section{Moral Corruption and Virtue in Posidonius's Thought. A Re-Examination of Some Fragments}

\author{
Giovanni ZaGo \\ Università di Firenze \\ giovanni.zago@unifi.it
}

Recibido: 01/10/2019 - Aceptado: 28/10/2020

DOI https://doi.org/10.20318/fons.2020.5054

\begin{abstract}
This article offers a text-critical and exegetical re-examination of some fragments of Posidonius on moral corruption and virtue (Sen. Ep. 90, 6 = Posidon. F 284 EK = F 448 Th; D.L. VII 91 = Posidon. F 29 EK = F 403 Th; D.L. VII $91=$ Posidon. F 2 EK = 435b Th), as well as of other related texts (Verg. Aen. VIII 313-328; Strab. XVI 2, 35-39= Posidon. F 133 Th; Sen. Ep. 89, 8). The author sheds new light on Posidonius's conception of the golden age (which can be reconstructed starting from Seneca's epistle 90) and on the text of Strab. XVI 2 , 35-39; proposes a new interpretation of Posidon. F 29 EK and an emendation to Posidon. F 2 EK; notices for the first time the Posidonian background of Sen. Ep. 89, 8.
\end{abstract}

Keywords. Posidonius, fragments, textual criticism, moral corruption, virtue

\section{Resumen}

Este artículo ofrece un reexamen crítico-textual y exegético de algunos fragmentos de Posidonio sobre la corrupción moral y la virtud (Sen. Ep. 90, 6 = Posidon. F 284 EK = F 448 Th; D.L. VII 91 = Posidon. F 29 EK = F 403 Th; D.L. VII 91 = Posidon. F 2 EK = 435b Th), así como de otros textos relacionados (Verg. Aen. VIII 313-328; Strab. XVI 2, 35-39 = Posidon. F 133 Th; Sen. Ep. 89, 8). El autor arroja nueva luz sobre la concepción posidoniana de la edad de oro (que puede reconstruirse a partir de la epístola 90 de Séneca) y sobre el texto de Strab. XVI 2, 35-39; propone una nueva interpretación de Posidon. F 29 EK y una enmienda a Posidon. F 2 EK; advierte por primera vez el trasfondo posidoniano de Sen. Ep. 89, 8.

Palabras clave: Posidonio, fragmentos, crítica textual, corrupción moral, virtud

Secondo lo Stoicismo ortodosso l'uomo nasce con una naturale predisposizione al

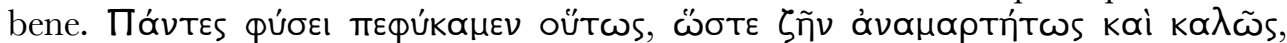
afferma Musonio Rufo (2, p. 6, 5-6 Hense) ${ }^{1}$ : «tutti per natura siamo conformati in modo tale da vivere senza peccato e virtuosamente». Il male si spiega, per gli Stoici,

${ }^{1}$ Cf. Sen. Ep. 94, 56: nulli nos vitio natura conciliat: illa integros ac liberos genuit.

ПНГН/FONS 5(2020), 103-114

ISSN 2445-2297 www.uc3m.es/pege
G. Zago, Corruzione morale e virtù in Posidonio DOI https://doi.org/10.20318/fons.2020.5054 
con la Sı๔отрофń, il pervertimento che distoglie l'individuo dal cammino verso la

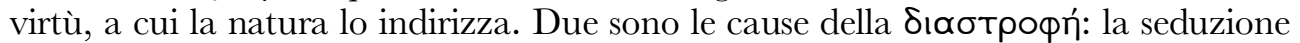

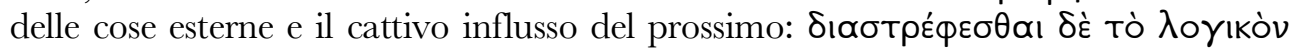

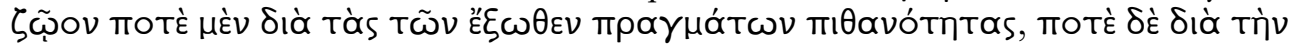

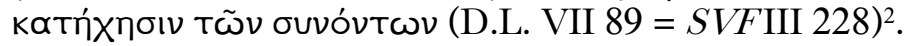

La dottrina ora sintetizzata sembra essere stata condivisa da Posidonio; la sua concezione circa l'origine delle passioni e del vizio è stata, infatti, recentemente dimostrata da Tieleman, in pagine a mio avviso persuasive, come de facto conforme a quella stoica-ortodossa ${ }^{3}$.

Vorrei concentrarmi, in questo contributo, su un punto non ancora messo a fuoco soddisfacentemente dalla critica, ossia sul ruolo essenziale nella storia politica e culturale del genere umano che Posidonio sembra avere assegnato tanto alla naturale

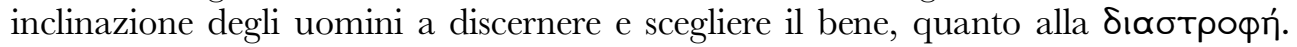
Cercherò di mostrare, infatti, che per Posidonio l' "età dell'oro' è il frutto della sinergia tra la perfetta virtù di alcuni membri di una comunità e la propensione al bene degli

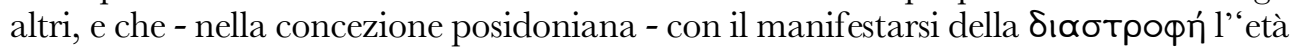
dell'oro' termina.

Come apprendiamo dall' Epistola 90 di Seneca, per Posidonio nella cosiddetta 'età dell'oro' (5: illo [...] saeculo quod aureum perhibent) gli uomini, che allora naturam incorrupti sequebantur (4), si sarebbero spontaneamente sottomessi agli individui intellettualmente superiori - individui che Posidonio definiva sapientes (lo Stoico

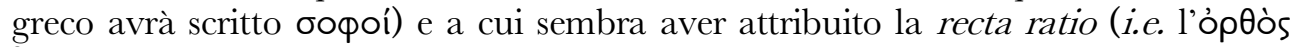
$\lambda$ óyos), considerandoli dunque, evidentemente, genuini sapienti ${ }^{4}$.

Tali individui sarebbero stati gli illuminati reges (reges per volontà dei sudditi) ${ }^{5}$ delle prime, 'auree', comunità umane politicamente organizzate, e avrebbero dato l'originario, fondamentale impulso allo sviluppo della civiltà, inventando le tecniche necessarie alla sopravvivenza del genere umano (Sen. Ep. 90, 7). Bisogna inferire che Posidonio riconduceva la spontanea sottomissione dei primi uomini ai sapientes da un lato alla sopra evocata tendenza naturale degli esseri umani al bene, dall'altro al loro connaturato istinto di autoconservazione ${ }^{6}$.

\footnotetext{
${ }^{2}$ Cf. SVF III 229-236; cf. TIELEMAN (2003), 132-139.

3 Si veda Tieleman (2003), 198-287 e passim. Secondo l'interpretazione convenzionale pretielemaniana - cf. e.g. BEES (2004), 46-74; 332-337 -, invece, Posidonio avrebbe attribuito all'uomo non solo una predisposizione al bene, ma anche un'innata inclinazione alla malvagità.

${ }^{4}$ Ep. 90, 5; 24-25. Sugli elementi posidoniani nell' Ep. 90 (lettera di eccezionale complessità) mi sia consentito di rimandare a un mio lavoro di qualche anno or sono (ZAGO, 2012), che mi piacerebbe, si vacabit, ripubblicare con modifiche (non minime) e supplementi. A mio parere l'opera posidoniana (perduta, come tutto Posidonio) cui Seneca ha attinto per comporre l' $E p .90$ era il Protrettico. Da vedere anche, sui segmenti posidoniani della lettera senecana (contestualizzati in un'agile e dotta storia del pensiero politico stoico), le fini considerazioni di WILDBERGER (2018), 124-133.

5 Nemo [scil. tra i sapientes reges dell'"età dell'oro'] quantum posset adversus eos experiebatur per quos coeperat posse (Ep. 90, 5).

${ }^{6}$ Secondo la dottrina stoica della oíkeícorıs l'uomo è geneticamente programmato per provvedere alla sua propria conservazione e a quella della specie. Il più rilevante contributo recente sulla dottrina
} 
È probabile che Posidonio non immaginasse uno Stato primordiale cosmopolita con al vertice una pluralità di re sapienti che governavano assieme (come nella Repubblica di Platone) ${ }^{7}$, ma piuttosto che pensasse a una molteplicità di sapientes reges, ciascuno dei quali avrebbe dominato su una singola comunità ${ }^{8}$, che lo avrebbe designato come guida e che attorno a lui si sarebbe sviluppata.

L'età dell'oro' evocata da Posidonio termina quando i vizi «sottentrano» e i regna si tramutano in tirannidi (Ep. 90, 6: subrepentibus vitiis in tyrannidem regna conversa sunt). L'esposizione senecana è fortemente brachilogica, e il testo non dice come si sia

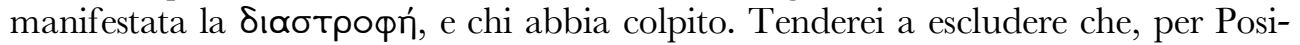
donio, siano stati i sapientes reges a tramutarsi in tiranni. Secondo Posidonio pienamente concorde, su questo punto, con l'ortodossia stoica -, i бoфoí sono infatti

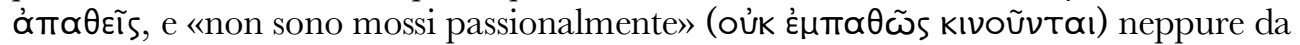
ciò a cui aspirano e che considerano il sommo bene, ossia la virtù'; impossibile, dunque, che i re dell'"età dell'oro' siano stati traviati dalla seduzione delle cose esterne o dal cattivo influsso del prossimo, e che, dopo essere stati sovrani ideali, si siano volti al vizio e abbiano ambito alla tirannide.

Ipotizzerei, se mai, che la corruzione si sia insinuata nei figli dei sapientes reges, alla morte dei padri ascesi al regno per volontà dei sudditi1 ${ }^{10}$. Non escluderei che Posidonio pensasse che con l'avvento dei figli al potere si fosse rotta l'armonia 'aurea' tra regnante e sottoposti, e che si fossero di conseguenza create le condizioni per la

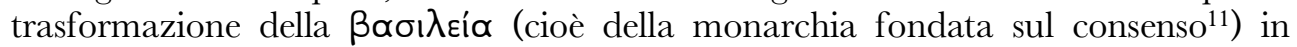
tirannide. Si confronti quel che Polibio scrive a proposito dei discendenti dei primitivi

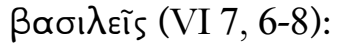

della oíkeíwoıs è BEes (2004); non condivido però l'interpretazione che Bees dà della psicologia posidoniana (cf. n. 3).

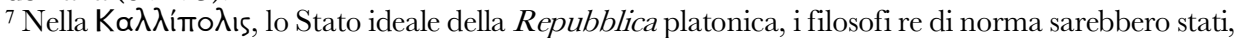
appunto, più d'uno.

${ }^{8} \mathrm{Se}$, come penso, Posidonio accettava l'idea stoica secondo la quale i primi uomini sarebbero nati come funghi in omnibus terris et agris (Lact. Inst. VII 4, 2-3), allora è del tutto verisimile che egli immaginasse comunità primordiali sparse per tutta l'ecumene.

${ }^{9}$ Cf. Gal. PHP IV 5, 26-27; 29; V 2, 5 De Lacy (Posidon. F 163-164 EK = F 409; 413 Th): cf. Roskam (2005), 51; 56. Con i sigla EK e Th faccio riferimento alle due fondamentali edizioni dei frammenti posidoniani, quella di Edelstein e Kidd (EDELSTEIN-KIDD, 1972) e quella di THEILER (1982).

${ }^{10}$ Per l'idea secondo cui la monarchia dei $\beta \alpha \sigma \iota \lambda \varepsilon \tau_{s}$ primitivi, amati per le loro benemerenze, sarebbe

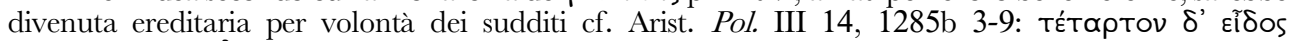

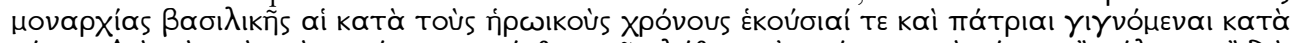

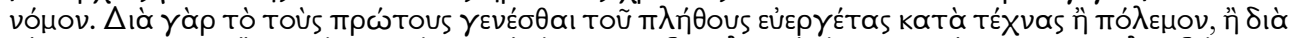

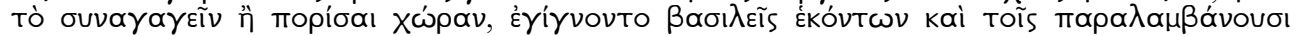
па́трıo,; Plb. VI 7, 2, ove si dice che i sottoposti avrebbero trasmesso il potere regio ai figli dei primi $\beta \alpha \sigma i \lambda \varepsilon \tilde{s}$, prevedendo che essi si sarebbero rivelati dotati delle stesse qualità morali e politiche dei padri:

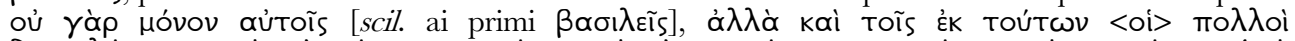

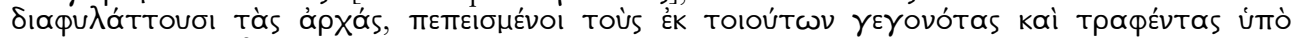

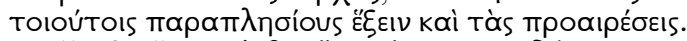

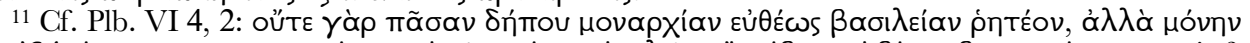

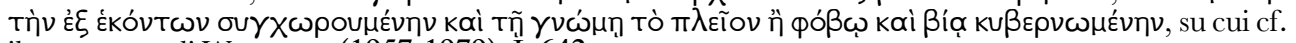
il commento di WALBANK (1957-1979), I, 642. 


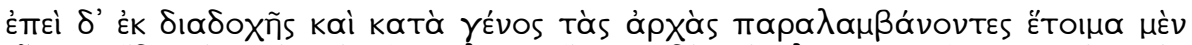

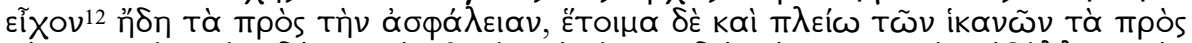

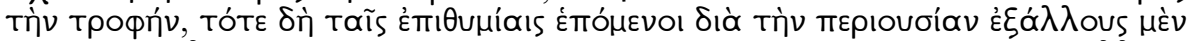

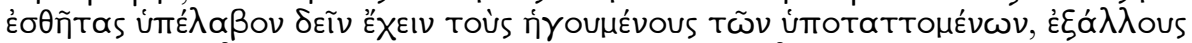

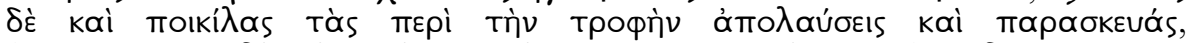

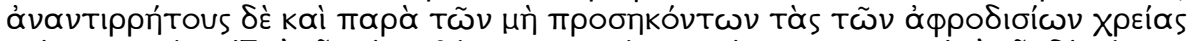

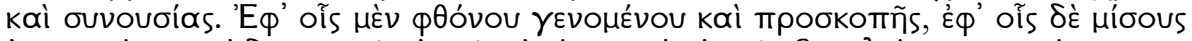
ÉKKaı

«Poiché, succedendo al potere sulla base della stirpe, avevano ormai pronti i mezzi di difesa, e pronti e anche superiori al necessario i viveri, allora, assecondando le proprie brame, a causa di tale sovrabbondanza ritennero che i governanti dovessero avere vesti diverse da quelle dei sudditi, e un'alimentazione peculiare, varia e gradevole, e che non dovessero subire dinieghi nel godimento dei piaceri sessuali e nelle relazioni erotiche, anche quelle illecite. Suscitarono così talora invidia e risentimento, talora odio infiammato e ira ostile, e dalla $\beta \alpha \sigma ı \lambda \varepsilon i ́ \alpha$ nacque la tirannide».

Più probabile, tuttavia, sembra un'altra ipotesi, ovvero che, secondo Posidonio, alla morte dei sapientes reges i nuovi monarchi avessero preso il potere non ék

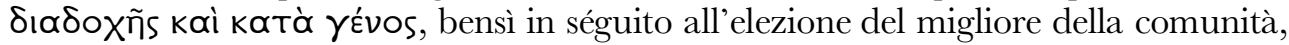
ma che gli eletti non fossero all'altezza del compito. In maniera analoga, ossia, appunto, in séguito alla scelta di governanti che sono i migliori tra gli eleggibili, ma che risultano indegni delle funzioni che sono destinati a ricoprire, si verifica la crisi dello Stato dei filosofi descritta nell'VIII libro della Repubblica platonica, e la degenerazione del suo regime politico (cf. Pl. $R$. VIII 545c ss.).

L'ipotesi che sto avanzando, secondo la quale l' età dell'oro' immaginata da

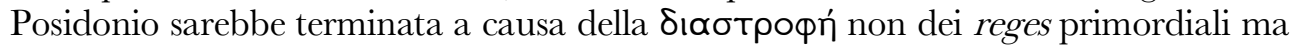
dei loro successori e delle comunità politiche di cui questi ultimi sarebbero stati emanazione, è suffragata, inoltre, da due passi di matrice posidoniana che trattano di 'età dell'oro' e di monarchia ideale. Il primo è Verg. Aen. 8, 313-328, ove Evandro afferma che Saturno, in qualità di re evergeta e civilizzatore, avrebbe instaurato nel Lazio un'epoca 'aurea', che si sarebbe poi conclusa a causa dell'insorgere dei vizi (non in Saturno, ma appunto tra i suoi sudditi) ${ }^{13}$.

Il secondo passo è Strab. XVI 2, 35-3914, in cui leggiamo che Mosè, sacerdote egizio, con la sua predicazione circa la reale natura della divinità (stoicamente con-

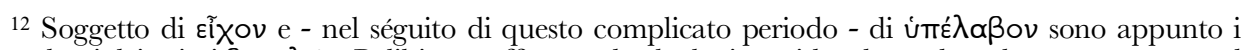

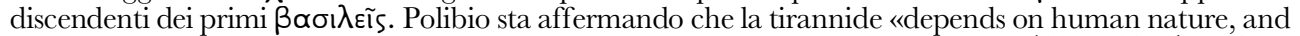
the vices which arise naturally in the 'second generation' in power»: cf. WALBANK (1957-1979), I, 655.

${ }^{13}$ Che il luogo virgiliano abbia ascendenza posidoniana fu intuito da Norden (1893), 425-428. Ho rilanciato e corroborato l'intuizione di Norden nella relazione Ancient Latium and the Golden Age. On the Source of Aen. 8, 313-336, che ho presentato al convegno Rome's Future, Rome's Past: the $8^{\text {th }}$ Book of the Aeneid (University of Manchester, 13-15 maggio 2019) e che presto pubblicherò.

${ }^{14}$ La matrice posidoniana del passo di Strabone è stata riconosciuta da vari illustri studiosi: segnalo in particolare REINHARDT (1928), THEILER (1982), che stampa il passo come Posidon. F 133, e RADT (2002-2011), VIII, 321, che offre ulteriore bibliografia. Il luogo straboniano non è invece accolto nell'edizione EK dei frammenti posidoniani (cf. n. 18). 


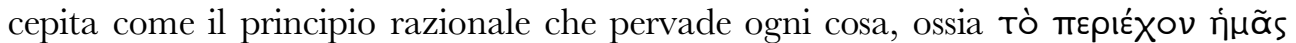

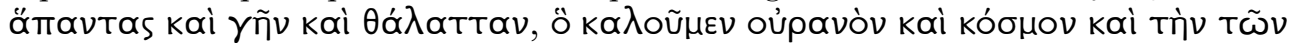

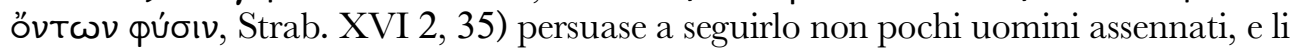
condusse nel luogo ove Gerusalemme sarebbe stata fondata:

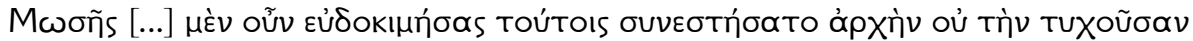

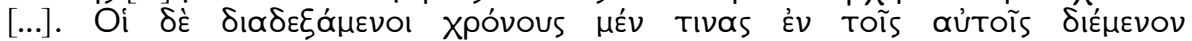

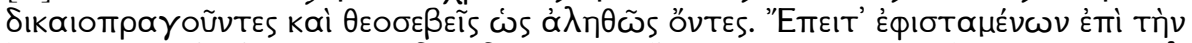

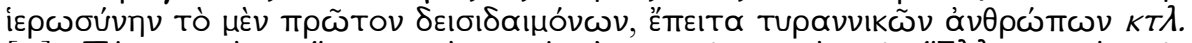

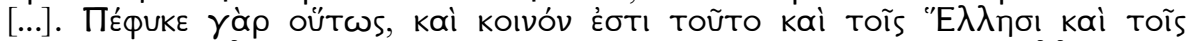

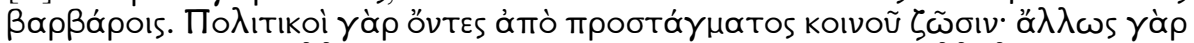

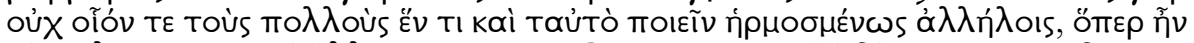

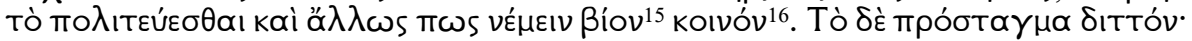

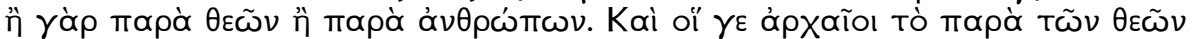

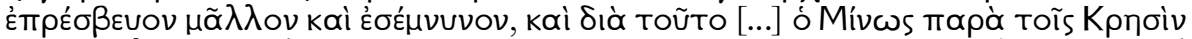

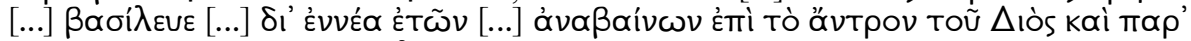

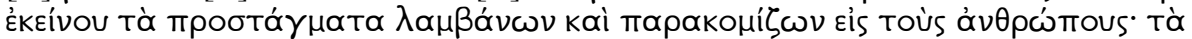

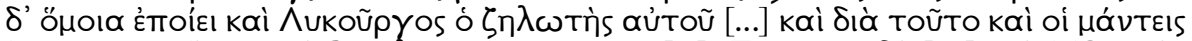

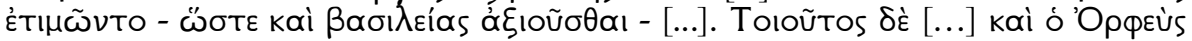

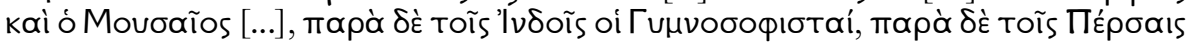

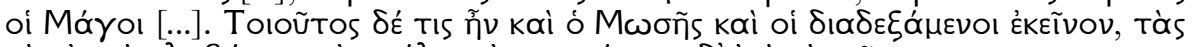

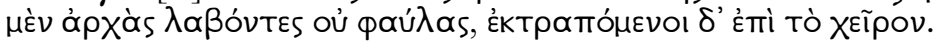

«Mosè $[. .$.$] con ciò acquisì dunque fama \mathrm{e}$ fondò un dominio non dappoco [...]. I suoi successori per un certo periodo si mantennero fedeli al suo esempio operando con giustizia ed essendo veramente pii. In séguito, essendo preposti al sacerdozio uomini dapprima superstiziosi, poi tirannici ecc. [...]. Ė naturale che sia così, e ciò è comune sia agli Elleni che ai barbari. In quanto esseri politici, infatti, essi vivono obbedendo a

${ }^{15}$ Come mi fa notare uno degli anonimi referee di Pegé/Fons, che ringrazio, Strabone (Posidonio?)

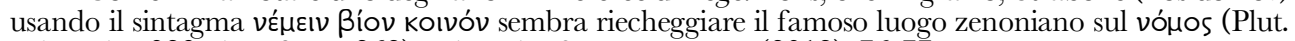
Virt. Alex. 329a-b = SVFI 262), sul quale cf. WILDBERGER (2018), 76-77.

${ }^{16}$ La mia interpunzione di quest'ultimo periodo (Strab. XVI 2,38) è diversa da quella vulgata, che sia TheILER (1982), F 133, sia l'ultimo editore critico di Strabone, RADT (2002-2011), IV, 342,

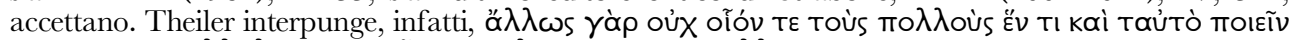

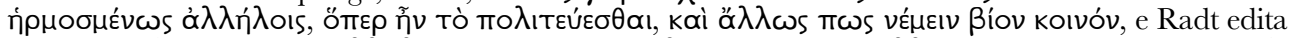

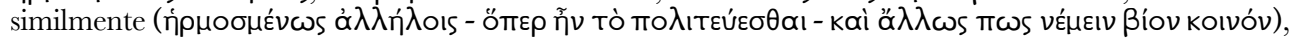
traducendo: «sonst ist es ja nicht möglich, daß die Menge in Einklang miteinander ein und dasselbe tut darin besteht doch das Leben in einem Staat - und einigermaßen ein gemeinsames Leben führt»; traduzioni analoghe in ReINHARDT (1928), 16; MALITZ (1983), 318; BAR-KochVA (2010), 360. A mio

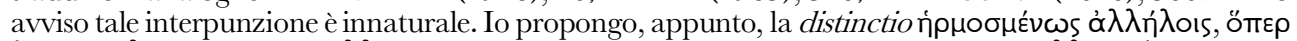

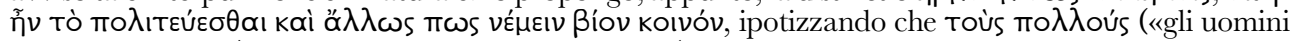
in massima parte») si riferisca agli Elleni e ai barbari (menzionati assieme nel periodo immediatamente

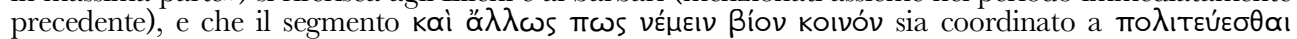

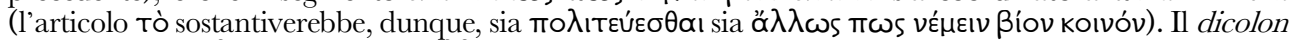

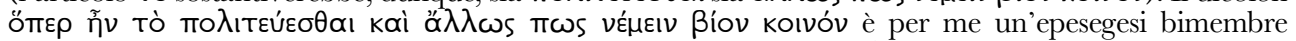

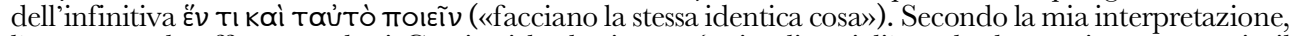
l'autore vuole affermare che i Greci e i barbari sono 'animali sociali', e che hanno in comune sia il

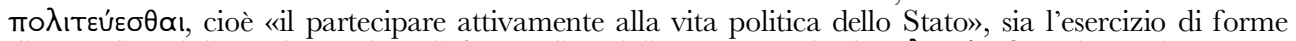

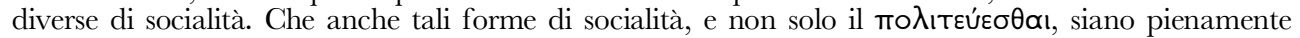

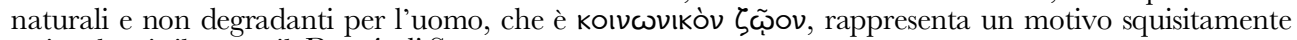
stoico: basti rileggere il De otio di Seneca. 
un comando comune: in caso contrario non sarebbe possibile, infatti, che gli uomini in massima parte facciano, all'unisono gli uni con gli altri, la stessa identica cosa, ovverosia che partecipino alla vita politica dello Stato e conducano in qualche altro modo un'esistenza comunitaria. Il comando è di duplice natura: viene dagli dèi oppure dagli uomini. E gli antichi attribuivano maggior prestigio e importanza a quello proveniente dagli dèi, e per questo [...] presso i Cretesi [...] regnava Minosse, che ogni nove anni [...] saliva all'antro di Zeus, e da Zeus riceveva i comandi e li comunicava agli uomini. E simili cose faceva anche Licurgo, il suo emulo [...]. E per questo anche gli indovini erano onorati, tanto da essere ritenuti degni del potere regale [...]. Tali [scil. erano] [...] Orfeo e Museo [...], e presso gli Indiani i Gimnosofisti, presso i Persiani i Magi [...]. Tali erano anche Mosè e i suoi successori, che dopo inizi non spregevoli degenerarono.

L'analogia tra il passo posidoniano-straboniano e Sen. Ep. 90, 4-6 appare palese. In entrambi i brani leggiamo che individui eccezionali e benefici, nati in più luoghi dell'ecumene, ricevono spontanea obbedienza e ottengono la leadership e la $\beta \propto \sigma ı \lambda \varepsilon i ́ a$, che però degenera in tirannide quando i loro successori prendono il potere. In entrambi i brani, insomma, viene spiegata allo stesso modo la genesi dello Stato ideale (e quindi, per così dire, di un"'epoca aurea'), genesi che, evidentemente, Posidonio riteneva possibile in ogni momento e in ogni luogo, a condizione che ci fosse un

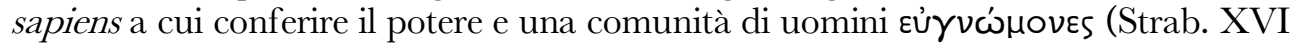
2, 36) o incorrupti (Sen. Ep. 90, 4), tali da riconoscerne l'eccellenza e attribuirgli il comando. Per Posidonio, sembra di capire, le 'età dell'oro' sono potenzialmente infinite; tutte però sono fisiologicamente destinate a concludersi a causa della Sıаотрофń, che cito subrepit ${ }^{17}$.

L'affinità tra Sen. Ep. 90, 4-6 e il passo di Strabone da un lato supporta fortemente la tesi secondo cui quest'ultimo avrebbe matrice posidoniana ${ }^{18}$, dall'altro stimola a utilizzare, come appunto io ho fatto, il brano straboniano per l'esegesi della pericope

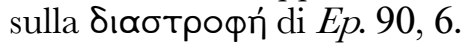

${ }^{17}$ Cf. Sen. QNIII 30, 8, un passo che forse risente dell'influsso della dottrina posidoniana circa l'"età aurea'. Sed de hoc alias.

${ }^{18}$ L'affinità non è percepita da KIDD (1988), II, 951-952, ad Posidon. F 279 EK, secondo cui in Strabone non ci sarebbe nulla di «caratteristicamente posidoniano» (e infatti Strab. XVI 2, 35-39 non figura nell'edizione EK dei frammenti di Posidonio). BAR-KochVA (2010), 358-362, considera posidoniani i paragrafi 35-37 di Strabone, non posidoniani i paragrafi 38-39, sulla base essenzialmente della differenza tra la lista di legislatori data nel luogo straboniano e quella posidoniano-senecana di $E p$. 90, 6. Si tratta, tuttavia, di un argomento inconsistente: Bar-Kochva non tiene in considerazione, infatti, che Seneca e Strabone attingono a due opere distinte: al Protrettico (o comunque a un'opera filosofica) Seneca (cf. n. 4), alle Storie - verisimilmente: cf. ThEILER (1982), II, 96-98 - Strabone. In due opere diverse Posidonio poteva ben aver proposto due liste diverse! Nettamente più significativa di questa divergenza è invece, a mio parere, l'affinità ideologica, che ho cercato di mettere in luce, tra Strab. XVI 2, 35-39 nel suo complesso e Sen. Ep. 90, 4-6, affinità che Bar-Kochva non può cogliere appieno perché fraintende completamente il passo senecano-posidoniano, immaginando a torto (BAR-KOCHVA, 2010, 367-368) che il regnum dei sapienti evocato da Posidonio fosse «a universal society encompassing all of mankind», e addirittura che «the sages are obviously [sid!] the ones through whom 'he (the king) had begun to have power' (Sen. Ep. 90, 5 fin.)», quasi che in Sen. Ep. 90, 4-6 fosse evocato un rex universale eletto da un comitato di saggi. Nella pericope di Ep. 90, 5 richiamata da Bar-Kochva e da noi trascritta supra, alla n. 5, per quos («through whom») si riferisce invece, come abbiamo detto, all'insieme dei membri delle singole comunità primordiali, ciascuna delle quali sceglie un sapiens come suo rex. 
Perché la ricostruzione della dottrina posidoniana in merito all' 'età dell'oro' che stiamo proponendo appaia verisimile dobbiamo tuttavia verificare se per Posidonio la virtù e la sapienza fossero obiettivi effettivamente raggiungibili per l'uomo. Credo si possa affermare con sicurezza che Posidonio le ritenesse tali. Un passo di Diogene Laerzio, in particolare, lo testimonia.

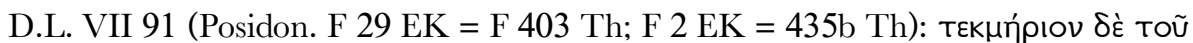

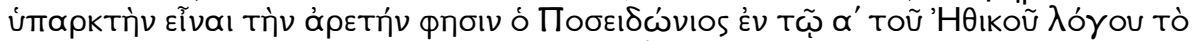

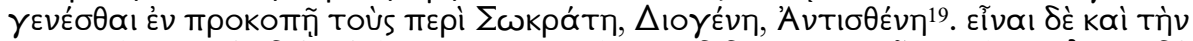

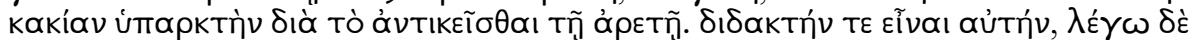

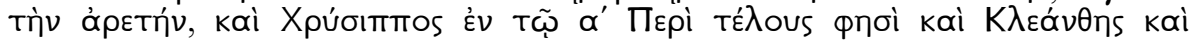

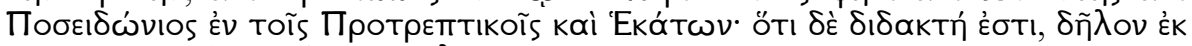

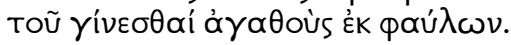

«Prova dell'esistenza della virtù Posidonio, nel primo libro dell'Etica, dice essere il progresso morale di Socrate, Diogene, Antistene. Afferma inoltre che anche il vizio esiste, in quanto antitetico alla virtù. E che essa, cioè la virtù, sia insegnabile lo sostengono sia Crisippo, nel primo libro dell'opera Sul fine, sia Cleante, sia Posidonio, nel Protrettico, sia Ecatone; che essa sia insegnabile è evidente dal fatto che si diventa buoni da individui dappoco che si era».

Il segmento laerziano, come si vede, contiene due frammenti di Posidonio

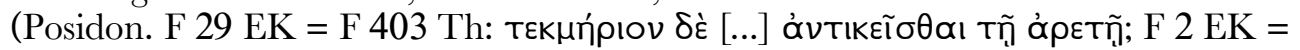

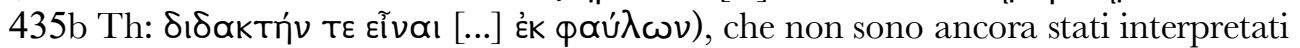
(ed emendati) in maniera soddisfacente. Iniziamo dal primo (Posidon. F 29 EK). Per Sedley ${ }^{20}$, questo passo significherebbe che, secondo Posidonio, Socrate, Diogene e Antistene avrebbero posseduto la virtù, e con il possederla ne avrebbero dunque dimostrato l'esistenza; se non l'avessero posseduta, infatti, essi non sarebbero stati tanto abili nel promuovere il progresso morale (трокоти́) dei loro allievi. La трокоти́ degli allievi dimostrerebbe, insomma, la ápeтŕ dei maestri, che a sua volta dimostrerebbe l'esistenza della ápetŕ tout court ${ }^{2}$. Tale interpretazione mi pare

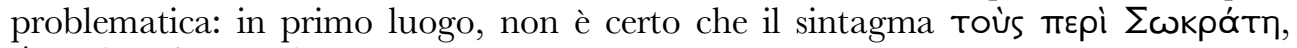

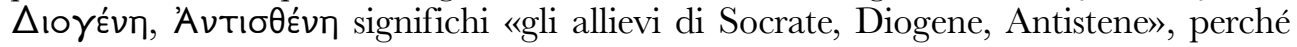
potrebbe anch'essere stato usato per indicare semplicemente «Socrate, Diogene, Antistene» (così, appunto, ho tradotto), oppure «Socrate, Diogene, Antistene e i loro

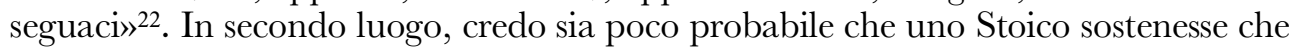
dal progresso morale degli allievi fosse inferibile la virtù dei maestri; dalla прокоти́ degli allievi uno Stoico avrebbe se mai desunto - credo - che проко́ттоvтєs («pro-

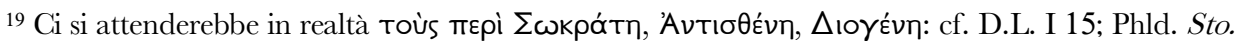
3, col. XIII, 1-4 DORANDI (1982). Suggerirei dunque, come congettura 'diagnostica', di trasporre, in

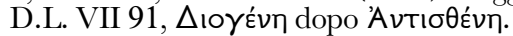

${ }^{20}$ SedLEy (2007), 233 n. 67. Sedley.

${ }^{21}$ L'interpretazione di Sedley è stata in parte anticipata da BONHÖFFER (1894), 217 n. 1, ignoto a

22 Sul significato del nesso oi mepì cf. Brouwer (2014), 107-109. 
gredienti») erano anche i maestri ${ }^{23}$. Mi sembra dunque opportuno proporre un'ipotesi alternativa, e mi pare che l'esegesi più naturale del frammento posidoniano sia un'altra, ossia che per Posidonio fosse la прокотп́ in se stessa a dimostrare l'esistenza della åotrí.

Nella concezione stoica, progredire moralmente significa dedicarsi alla filosofia e intraprendere un cammino che conduce alla virtù portando l'uomo a perfezionare la sua naturale capacità di discernere il bene; chi progredisce verso la virtù, ma non è ancora giunto alla meta, continua, tuttavia, ad appartenere al novero dei qaũ $\lambda$ o (ossia degli «uomini dappoco», degli «stolti»). Con una dotta similitudine, Cleante (Ar.Did. apud Stob. II 7, $5^{\mathrm{b} 8}=$ SVFI 566) paragonava gli esseri umani ai meliambi,

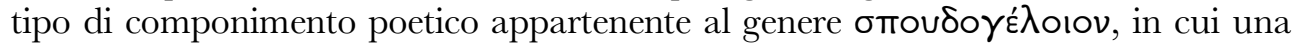
forma apparentemente 'bassa' e leggera ( $\phi$ aú $\lambda \eta)$ era usata per trasmettere un inse-

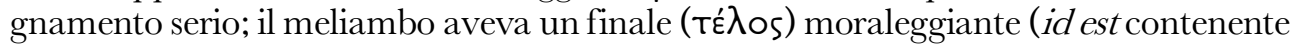
un $\sigma \omega ф p o v i \sigma \mu o ́ s)$, che chiariva e dava senso al componimento stesso, il quale - se

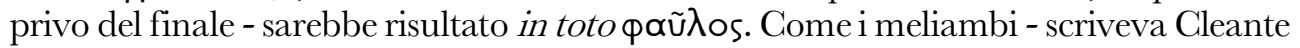

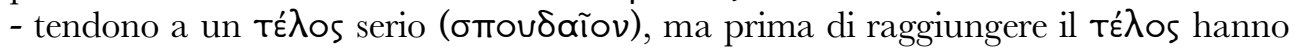
un carattere leggero e 'basso', così gli uomini tendono naturalmente alla virtù, ma finché non l'hanno ottenuta sono stolti ( Qũ̃

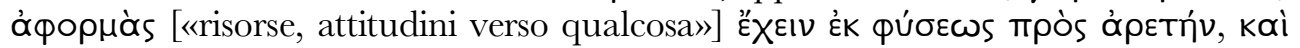

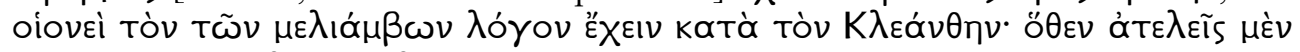

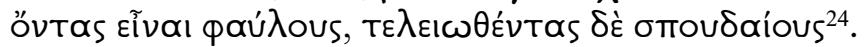

La dottrina posidoniana di cui ci informa Diogene Laerzio in VII 91 (Posidon. F $29 \mathrm{EK}$ ), se interpretata come io propongo, potrebbe dunque sembrare paradossale; nondimeno, ritengo che la mia interpretazione sia sostenibile, non solo perché è l'esegesi più naturale del luogo posidoniano-diogeniano, ma anche per ragioni filosofiche. L'argomento di cui Posidonio si serviva, teste Diogene, per dimostrare l'esistenza del vizio era di natura logica (il vizio esiste in quanto antitetico alla virtù) ${ }^{25}$; l'argomento con cui dimostrava l'esistenza della virtù era empirico (l'esistenza reale della трокотп́), ma - credo - con implicazioni epistemologiche. È probabile infatti, a mio avviso, che Posidonio sviluppasse questa sua prova dell'esistenza della virtù prendendo il paradosso di Menone come assunto: se non si può cercare quello che non si conosce (Pl. Men. 80d 5-e 5), allora chi ricerca la virtù deve sapere che cosa sia la virtù stessa; se la прокотń è reale (ossia è un reale progresso in direzione della ápetŕ)

${ }^{23}$ Cf. l'Ep. 6 di Seneca, in cui il merito del progresso morale di Metrodoro, Ermarco e Polieno viene attribuito al contubernium Epicuri (6); è chiaro che, nella prospettiva senecana, la grandezza degli allievi di Epicuro, definiti magni viri, ma certo lontani dalla perfetta sapienza, dimostra che Epicuro stesso era

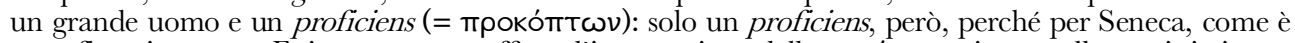
superfluo rimarcare, Epicuro non era affatto l'incarnazione della ooфía genuina, quella a cui si giunge avendo compiuto interamente la прокоти́ (cf. infra). Per gli Stoici - è fin troppo noto - il sapiens è l'uomo perfetto, dotato di ogni qualità, infallibile e per nulla inferiore a Giove: cf. ZAGO (2017) e, in generale sul бoфós stoico, BROUWER (2014).

${ }^{24}$ Sul testo e il senso di questo passo cf. Livrea (1987); Zago (2012), 32-33 e n. 53. Sul tema del progresso morale, nello Stoicismo e non, cf. RoskaM (2005), ottimamente documentato.

${ }^{25}$ Per tale argomento cf. Pl. Tht. 176a; SVF II 1169; cf. KIDD (1988), II, i, 153. 


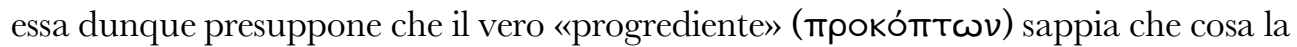
virtù sia realmente, e questo implica che la virtù sia qualcosa di reale, altrimenti neppure la трокотп́ lo sarebbe. La mia interpretazione mi pare peraltro supportata da un parallelo, ovvero Sen. Ep. 89, 4-8:

«Sapientia perfectum bonum est mentis humanae; philosophia sapientiae amor est et adfectatio: haec eo tendit quo illa pervenit. Philosophia unde dicta sit apparet: ipso enim nomine fatetur quid amet. [5] Sapientiam quidam ita finierunt ut dicerent divinorum et humanorum scientiam; quidam ita: sapientia est nosse divina et humana et horum causas. Supervacua mihi haec videtur adiectio, quia causae divinorum humanorumque pars divinorum sunt. Philosophiam quoque fuerunt qui aliter atque aliter finirent: alii studium illam virtutis esse dixerunt, alii studium corrigendae mentis; a quibusdam dicta est adpetitio rectae rationis. [6] Illud quasi constitit, aliquid inter philosophiam et sapientiam interesse: neque enim fieri potest ut idem sit quod adfectatur et quod adfectat [...]. [8] Quidam ex nostris [scil. tra gli Stoici], quamvis philosophia studium virtutis esset et haec peteretur, illa peteret, tamen non putaverunt illas distrahi posse: nam nec philosophia sine virtute est nec sine philosophia virtus. Philosophia studium virtutis est, sed per ipsam virtutem; nec virtus autem esse sine studio sui potest nec virtutis studium sine ipsa [...]: ad virtutem venitur per ipsam, cohaerent inter se philosophia virtusque».

«La sapienza è il bene della mente umana portato a perfezione; la filosofia è amore e aspirazione alla sapienza: essa tende alla meta cui la sapienza è pervenuta. Risulta evidente quale sia l'origine del termine filosofia: nel nome stesso rivela ciò che ama. [5] Alcuni definirono in tal modo la sapienza: conoscenza delle cose divine e di quelle umane; altri così: sapienza è conoscere le cose divine, quelle umane e le loro cause. Questa aggiunta mi sembra superflua, dal momento che le cause delle cose divine e di quelle umane sono parte delle cose divine. Ci sono stati uomini che hanno definito anche la filosofia in modi diversi: alcuni la hanno chiamata ricerca ${ }^{26}$ della virtù, altri ricerca finalizzata a rendere retta la mente; da qualcuno essa è stata definita appetizione della retta ragione. [6] Ciò, per così dire, rappresentò un punto fermo, che tra filosofia e sapienza ci sia una qualche differenza: non può accadere, infatti, che ciò a cui si aspira e ciò che aspira siano la stessa cosa [...]. [8] Alcuni tra i nostri [scil. gli Stoici], benché la filosofia sia ricerca della virtù e la virtù sia cercata, la filosofia cerchi, non reputarono, tuttavia, che esse fossero separabili: infatti, né la filosofia esiste senza virtù, né la virtù senza filosofia. La filosofia è ricerca della virtù, ma attraverso la virtù stessa; e d'altra parte non può esserci virtù senza ricerca di virtù, né ricerca di virtù senza la virtù stessa [...]: si giunge alla virtù attraverso la virtù stessa, filosofia e virtù sono intimamente legate».

Nessuno ha notato l'analogia tra il paragrafo 8 della lettera senecana e D.L. VII 91 (Posidon. F 29 EK), ma a mio parere è chiaro che per Posidonio, così come per quegli Stoici (quidam ex nostris) lì evocati da Seneca, ad virtutem venitur per ipsam

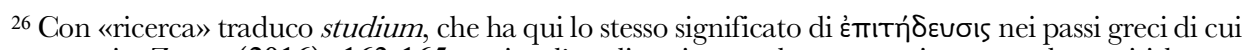
ho trattato in ZAGO (2016), 163-165, ossia «l'applicarsi a qualcosa per giungere ad acquisirlo e a possederlo compiutamente». 
(«si giunge alla virtù attraverso ${ }^{27}$ la virtù stessa»): il percorso filosofico (i.e. la трокотท́) che conduce alla ápєтŕ presuppone, cioè, l'esistenza della ápєtŕ stessa. Dal momento che non conosciamo nessun altro Stoico che abbia espresso tale idea dovremo, fino a prova contraria, attribuire matrice posidoniana a Sen. $E p .89,8^{28}$, e non potremo non vedere nel luogo senecano una delle chiavi di volta per l'interpretazione di D.L. VII 91 (Posidon. F 29 EK) ${ }^{29}$.

Veniamo ora al secondo frammento, D.L. VII 91 (Posidon. F 2 EK), da cui apprendiamo che per Posidonio la virtù è insegnabile. Quello che sopra ho trascritto e tradotto è il testo tràdito dai testimoni di Diogene Laerzio, che è conservato dal più recente editore dell'opera diogeniana, Dorandi ${ }^{30}$, ma che risulta problematico, e tale

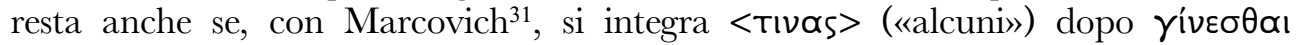
(traduzione: «è evidente dal fatto che <alcuni> diventano buoni da individui dappoco che erano»). Come osserva Kidd ${ }^{32}$, il divenire virtuosi non prova di per sé che la virtù sia insegnabile, perché si potrebbero postulare anche altre cause del fenomeno: la dimostrazione è dunque cattiva, e né Posidonio né altri Stoici l'avrebbero potuta proporre nella forma in cui i manoscritti di Diogene ce la tramandano. Kidd definisce, pertanto, «convincente», e registra nell'apparato critico della sua edizione del passo (Posidon. F 2 EK) l'ipotesi di Sandbach secondo la quale all'interno del segmento $\delta \tilde{n} \lambda \circ v[. ..] \phi a u ́ \lambda \omega \nu$ («è evidente [...] individui dappoco che si era») sarebbe caduto il participio $\delta \_\delta \alpha \sigma k o \mu \varepsilon ́ v o u s$ («ricevendo insegnamenti»). La proposta di Sandbach ${ }^{33}$ è senza dubbio intelligente (ma si potrebbe pensare anche alla caduta di sostantivi

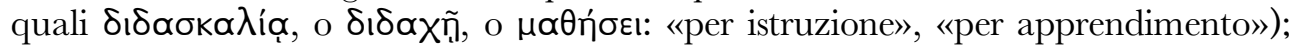
suggerirei, tuttavia, una soluzione diversa, più verisimile dal punto di vista della genesi

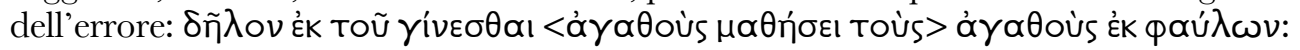
«è evidente dal fatto che $\mathrm{i}$ buoni diventano buoni per apprendimento da individui

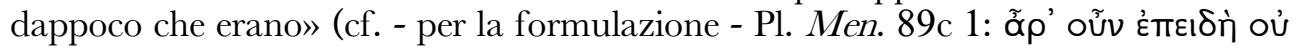

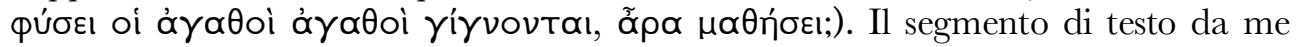

${ }^{27}$ Un referee mi fa rilevare che attribuendo a per ipsam un'accezione strumentale («per mezzo di») si potrebbe intendere il brano senecano come Sedley intende Posidon. F 29 EK: «si giunge alla virtù per mezzo della virtù stessa (scil. quella dei maestri)». L'osservazione è acuta, ma il contesto induce decisamente a propendere per l'interpretazione spaziale di per. $\mathrm{cf}$. venitur e, poco prima, l'immagine degli itinera quae ad urbes perducunt, che occorre in un segmento di $E p .89,8$ che non ho citato e che è lacunoso - cf. l'apparato di REYNOLDS (1965) -, ma il cui senso generale è chiaro.

${ }^{28}$ La locuzione senecana quidam ex nostris può senz'altro alludere a Posidonio o a suoi discepoli.

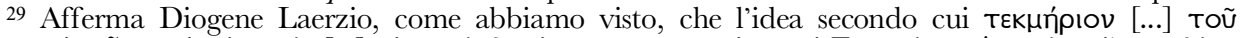

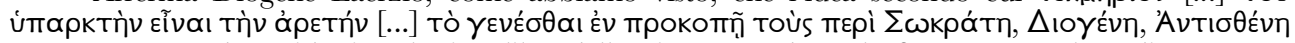
era stata esposta da Posidonio nel primo libro dell' Etica. Non è detto che Seneca avesse letto direttamente l'Etica di Posidonio; potrebbe infatti aver attinto a una Mittelquelle dossografica. Da un testo dossografico post-posidoniano sembrano dipendere - si noti - i paragrafi immediatamente seguenti (913) dell'ottantanovesima lettera senecana: cf. WILDBERGER (2006), I, 134. Interessante osservare che il paradosso di Menone è ricordato da Roskam (2005), 95-97, nella sua analisi di Sen. Ep. 89, 8. Roskam ignora, però, la connessione tra il passo di Seneca e D.L. VII 91 (Posidon. F 29 EK).

30 DORANDI (2013), 528.

31 Marcovich (1999), I, 498.

${ }^{32}$ KIDD (1988), II, i, 100.

${ }^{33}$ Citata in apparato, assieme all'integrazione di Marcovich, anche da DoRANDi (2013), 528. 
integrato potrebbe facilmente essere caduto per saut du même au même. Non si può, tuttavia, essere certi che la lacuna da noi ipotizzata sia un guasto occorso nella tradizione manoscritta di Diogene Laerzio, perché l'omissione potrebbe essersi verificata nella fonte cui Diogene attinge. Diogene, infatti, nell'elaborare il suo VII libro si rifaceva a compilazioni e dossografie, non ai testi originali di Crisippo, Cleante, Posidonio, Ecatone e degli altri Stoici ${ }^{34}$, e tendeva a prendere per buono ciò che in tali compilazioni trovava scritto (da qui tante stranezze e incongruenze). L'omissione di

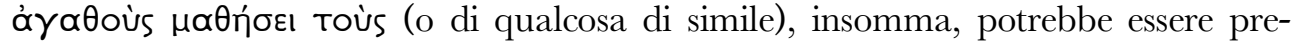
diogeniana e aver già sfigurato la fonte di Diogene. Non inserirei, dunque, nel testo di una nuova edizione di Diogene Laerzio la mia integrazione, ma la relegherei, 'diagnosticamente', in apparato; la porrei nel testo, invece, se dovessi rieditare i frammenti di Posidonio.

\section{Bibliografia}

Bar-Kochva (2010):

Bar-Kochva, B., The Image of the Jews in Greek Literature. The Hellenistic Period, BerkeleyLos Angeles-London.

Bees (2004):

Bees, R., Die Oikeiosislehre der Stoa, I, Rekonstruktion ihres Inhalts, Würzburg.

Bonhöffer (1894):

Bonhöffer, A., Die Ethik des Stoikers Epictet, Stuttgart.

Brouwer (2014):

Brouwer, R., The Stoic Sage. The Early Stoics on Wisdom, Sagehood and Socrates, Cambridge.

Dorandi (1982):

Dorandi, T., «Filodemo, Gli Stoici (PHerc. 155 e 339)», CErc XII, 91-133.

Dorandi (2013):

Dorandi, T., Diogenes Laertius. Lives of Eminent Philosophers, Cambridge.

Edelstein-Kidd (1972):

Edelstein, L., Kidd, I.G., Posidonius, I, The Fragments, Cambridge.

Hahm (1992):

Hahm, D.E., «Diogenes Laertius VII: On the Stoics», $A N R W$ II, 36, 6, 4076-4182.

Kidd (1988):

Kidd, I.G., Posidonius, II, i-ii, The Commentary, Cambridge.

Livrea (1987):

Livrea, E., «Un frammento di Cleante ed i meliambi di P. Oxy. 1082», ZPE LXVII, 37-41. Malitz (1983):

\footnotetext{
${ }^{34}$ Il problema delle fonti del VII libro diogeniano è straordinariamente complesso, e non a torto il libro è stato definito «a literary enigma» (HAHM, 1992, 4077). Che Diogene non avesse conoscenza diretta di opere posidoniane è sicuro: cf. KIDD (1988), II, i, 53-54, ad Posidon. T 66 EK; VerbeKE (1986).
} 
Malitz, J., Die Historien des Poseidonios, München.

Marcovich (1999):

Marcovich, M., Diogenis Laertii Vitae Philosophorum, I-II, Stutgardiae et Lipsiae.

Norden (1893):

Norden, E., «Beiträge zur Geschichte der griechischen Philosophie», JKPh, Suppl. 19, 365460.

Radt (2002-2011):

Radt, S., Strabons Geographika, I-X, Göttingen.

Reinhardt (1928):

Reinhardt, K., Poseidonios über Ursprung und Entartung; Heidelberg.

Reynolds (1965):

Reynolds, L.D., Senecae Ad Lucilium Epistulae morales, I-II, Oxonii.

Roskam (2005):

Roskam, G., On the Path to Virtue. The Stoic Doctrine of Moral Progress and its Reception in (Middle-)Platonism, Leuven.

Sedley (2007):

Sedley, D.N., Creationism and its Critics in Antiquity, Berkeley-Los Angeles-London.

Theiler (1982):

Theiler, W., Poseidonios, Die Fragmente, I-II, Berlin-New York.

Tieleman (2003):

Tieleman, T., Chrysippus 'On Affections. Reconstruction and Interpretation, Leiden-Boston. Verbeke (1986):

Verbeke, G., «Panétius et Posidonius chez Diogène Laërce», Elenchos VII, 103-131.

Walbank (1957-1979):

Walbank, F.W., A Historical Commentary on Polybius, I-III, Oxford.

Wildberger (2006):

Wildberger, J., Seneca und die Stoa. Der Platz des Menschen in der Welt, I-II, Berlin-New York.

Wildberger (2018):

Wildberger, J., The Stoics and the State, Baden-Baden.

Zago (2012):

Zago, G., Sapienza filosofica e cultura materiale. Posidonio e le altre fonti dell'Epistola 90 di Seneca, Bologna.

Zago (2016):

Zago, G., «Cinque note testuali a quattro prosatori», Prometheus XLII, 163-170.

Zago (2017):

Zago, G., «Due note sul testo e la fortuna di Orazio», Prometheus XLIII, 112-114. 